\title{
Structural Integration as an Adjunct to Outpatient Rehabilitation for Chronic Nonspecific Low Back Pain: A Randomized Pilot Clinical Trial
}

\author{
Eric E. Jacobson, ${ }^{1}$ Alec L. Meleger, ${ }^{2,3}$ Paolo Bonato, ${ }^{2,4,5}$ Peter M. Wayne, ${ }^{6}$ \\ Helene M. Langevin, ${ }^{6,7}$ Ted J. Kaptchuk, ${ }^{8}$ and Roger B. Davis ${ }^{8}$ \\ ${ }^{1}$ Department of Global Health \& Social Medicine, Harvard Medical School, 641 Huntington Avenue, Boston, MA 02115, USA \\ ${ }^{2}$ Department of Physical Therapy \& Rehabilitation, Harvard Medical School, Spaulding Rehabilitation Hospital, 300 First Avenue, \\ Charleston, MA 02129, USA \\ ${ }^{3}$ Spine Center, Newton-Wellesley Hospital, 159 Wells Avenue, Newton, MA 02459, USA \\ ${ }^{4}$ Motion Analysis Laboratory, Department of Physical Therapy \& Rehabilitation, Spaulding Rehabilitation Hospital, 300 First Avenue, \\ Charleston, MA 02129, USA \\ ${ }^{5}$ Harvard-MIT Division of Health Sciences and Technology, 45 Carleton Street, Cambridge, MA 02142, USA \\ ${ }^{6}$ Osher Center for Integrative Medicine, Division of Preventive Medicine, Harvard Medical School and Brigham and Women's Hospital, \\ 900 Commonwealth Avenue, Boston, MA 02215, USA \\ ${ }^{7}$ Department of Neurological Sciences, College of Medicine, University of Vermont, 89 Beaumont Avenue, Burlington, VT 05401, USA \\ ${ }^{8}$ Division of General Medicine \& Primary Care, Harvard Medical School and Beth Israel Deaconess Medical Center, \\ 330 Brookline Avenue, Boston, MA 02215, USA
}

Correspondence should be addressed to Eric E. Jacobson; eric_jacobson@hms.harvard.edu

Received 7 November 2014; Revised 8 January 2015; Accepted 8 January 2015

Academic Editor: Jenny M. Wilkinson

Copyright (C) 2015 Eric E. Jacobson et al. This is an open access article distributed under the Creative Commons Attribution License, which permits unrestricted use, distribution, and reproduction in any medium, provided the original work is properly cited.

Structural Integration (SI) is an alternative method of manipulation and movement education. To obtain preliminary data on feasibility, effectiveness, and adverse events (AE), 46 outpatients from Boston area with chronic nonspecific low back pain (CNSLBP) were randomized to parallel treatment groups of SI plus outpatient rehabilitation (OR) versus OR alone. Feasibility data were acceptable except for low compliance with OR and lengthy recruitment time. Intent-to-treat data on effectiveness were analyzed by Wilcoxon rank sum, $n=23$ per group. Median reductions in VAS Pain, the primary outcome, of $-26 \mathrm{~mm}$ in SI + OR versus 0 in OR alone were not significantly different $(P=0.075)$. Median reductions in RMDQ, the secondary outcome, of -2 points in SI + OR versus 0 in OR alone were significantly different $(P=0.007)$. Neither the proportions of participants with nor the seriousness of AE were significantly different. SI as an adjunct to OR for CNSLBP is not likely to provide additional reductions in pain but is likely to augment short term improvements in disability with a low additional burden of AE. A more definitive trial is feasible, but OR compliance and recruitment might be challenging. This trial is registered with ClinicalTrials.gov (NCT01322399).

\section{Introduction}

Chronic low back pain is among the most burdensome of health problems in prevalence and cost of care [1]. It is the leading cause of years lived with disability worldwide and the most frequent cause of functional loss in high-income countries $[2,3]$. Much of the economic burden is expended on costly surgical and rehabilitative services. Up to onethird of acute low back pain cases may become chronic and lead to disability [4]. In a majority of chronic cases (estimated at 85-95\%) a definitive diagnosis, that is, infection, neoplasm, osteoporosis, arthritis, fracture, radiculopathy, or inflammatory rheumatic processes, is ruled out, and these are designated as chronic "uncomplicated," "mechanical," 
or "nonspecific" low back pain (CNSLBP) [5]. There is no consensus on the optimal approach to the treatment of CNSLBP. Management typically includes some combination of analgesic or anti-inflammatory medication, directed therapeutic exercise, manipulation, cognitive-behavioral therapy, and patient education [6]. Systematic reviews have generally concluded that the benefits of these approaches are limited and mostly short-lived [7-11]. A large survey in the United States found that $54 \%$ of patients with low back or neck pain used complementary therapies and that approximately onethird of all visits to alternative care practices were for back or neck pain [12]. Low back pain has been reported to be the primary complaint in $40 \%$ of all visits to chiropractors, $20 \%$ to massage therapists, and 15\% to acupuncturists [13].

Structural Integration (SI) is an alternative manual therapy that is increasingly available and sometimes resorted to for the treatment of chronic musculoskeletal pain and disability. Developed by the biochemist Ida Rolf outside of orthodox medical science, it has been propagated as an alternative therapy since the mid-1950s. A few preliminary studies of low quality with small samples suggest effectiveness for musculoskeletal pain, but aside from a single case report, no clinical studies of SI for CNSLBP have been published to date [14-16]. The musculoskeletal pain studies and preliminary evidence regarding a number of hypothesized therapeutic mechanisms have been reviewed elsewhere [17].

The experience of SI treatment sometimes involves notable discomfort which has led to a reputation of being excessively painful and even to concerns as to its safety [18]. This has been a barrier to a more widespread adoption by conventional clinical services, although SI was successfully incorporated into at least one $[15,19]$. Despite these concerns, published data on adverse events (AE) associated with SI are limited to a single case and a small prospective case series $[20,21]$.

This study was designed to collect preliminary data on the feasibility, effectiveness, and AE associated with SI as an adjunct to outpatient rehabilitation (OR) versus $\mathrm{OR}$ alone for CNSLBP. The outcomes will inform the design of a more adequately powered clinical trial. We hypothesized that we could recruit and retain qualified participants who would comply with treatment regimens and data collection, that a course of SI + OR would improve low back related pain and disability significantly more than OR alone, and that SI could be delivered with acceptable levels of AE.

\section{Methods}

2.1. Design. Following baseline data collection, participants were randomized $1: 1$, and open label to parallel treatment groups. Follow-up data were collected at 20 weeks after baseline.

2.2. Setting, Recruitment, and Enrollment. The study was conducted at the Motion Analysis Laboratory (MAL), Spaulding Rehabilitation Hospital, Partners HealthCare, LLC, Boston, and was approved by the Spaulding institutional review board (IRB) (2010-P-00004). An independent monitor and a datamonitoring group provided additional oversight. The study was registered with ClinicalTrials.gov (NCT01322399) prior to beginning recruitment.

We included men and women aged 18-65 residing in the greater Boston area, with CNSLBP of $\geq 6$ months duration not attributed to infection, neoplasm, severe radiculopathy (assessed by frequent severe pain radiating down a leg), fracture, or inflammatory rheumatic process, with a patientrated bothersomeness of pain on average over the preceding 6 months $\geq 3$ on an 11-point ordinal scale $(0=$ none, $10=$ worst imaginable), that is, moderate to severe range. Prior arrangement to enter or having recently entered treatment at any Boston area outpatient rehabilitation clinic was also required. These and other criteria are given as follows.

\section{Enrollment Criteria}

\section{Inclusionary}

(i) Men and women aged 18-65,

(ii) CNSLBP of $\geq 6$ months duration, not attributed to infection, neoplasm, severe radiculopathy (as indicated by frequent severe pain radiating down a leg), fracture, or inflammatory rheumatic process,

(iii) self-rated bothersomeness of back pain self-rated on average over the preceding 6 months $\geq 3$ on an 11-point ordinal scale $(0=$ none, $10=$ worst imaginable $)$,

(iv) prior arrangement to enter a course of outpatient physical therapy for low back pain at a Boston area rehabilitation clinic,

(v) English language fluency and mental capacity sufficient to provide informed consent and participate in the study.

\section{Exclusionary}

(i) Impaired hearing, speech, vision, and mobility sufficient to interfere with participation in the study,

(ii) current or anticipated receipt of payments from Worker's Compensation or other insurance for disability attributed to low back pain,

(iii) prior treatment with any SI therapy (any variety, including structural massage),

(iv) plans to initiate additional treatment for back pain during the period of the study other than outpatient rehabilitation care, particularly massage or other manual therapies (e.g., chiropractic or osteopathic manipulation),

(v) exclusions for safety: unresolved musculoskeletal pathology of the lower limbs, current pregnancy, any implanted medical device (e.g., cardiopacemaker, shunts), osteoporosis, any hypercoagulation condition, eczema, skin infection, deep vein thrombosis, burns or other acute trauma including unhealed bone fractures or open wounds, psoriasis, psychiatric illness not well controlled, or current episode of exacerbated major depressive disorder, 
(vi) exclusions for anticipated lack of therapeutic response: severe radiculopathic pain, prior discectomy or implantation of rods, screws or plates, or fibromyalgia,

(vii) conditions that might confound therapeutic response or outcomes: medication with coumadin or prednisone, chronic steroid medication, daily use of narcotic analgesics, or alcohol or substance abuse,

(viii) conditions that might confound biomechanical data: current diagnosis of balance problems due to vestibular or other neurological impairments, severe or progressive neurological deficits including neuromotor impairment, estrogen supplementation, tricyclic antidepressants (if not on a regular steady dose at least one month prior to enrollment), or any substance that could impair balance,

(ix) conditions that would confound data on inflammatory biomarkers: type I diabetes, Crohn's disease, lupus, inflammatory bowel disease, ulcerative colitis, any other autoimmune disease, cancer, or body mass index $\geq 40$,

(x) any other major medical condition that has not been stabilized or that would impair the patient's ability to complete study activities.

Candidates were recruited through notices posted at Spaulding outpatient rehabilitation clinics and through a publically accessible online registry of interest in clinical studies [22]. The PI (Eric E. Jacobson) screened candidates and enrolled those that met entry criteria. They then proceeded immediately to baseline data collection. The study paid parking expenses and remunerated participants $\$ 50$ each time they visited the MAL for screening or data collection.

2.3. Randomization, Allocation, and Blinding. The study biostatistician (Roger B. Davis) generated randomization sequences in permutated blocks of 6 stratified by gender and sealed the individual assignments in two series of sequentially numbered opaque envelopes, which were color-coded by gender to allow for stratified allocation. These were stored in a locked metal file cabinet to which the investigators (Eric E. Jacobson, Alec L. Meleger, Paolo Bonato, Roger B. Davis) had access only to respond to emergent, severe $\mathrm{AE}$ and to complete IRB required reports. All investigators were initially blind to treatment assignment, but maintenance of blinding proved to be infeasible due to limitations of study staffing. A MAL staff member who had no other role in the study did have access in order to perform randomized allocation, which was done following each participant's baseline data collection. Neither the participants themselves nor the study therapists were blinded due to obvious differences between SI treatment and outpatient physical therapy (OR).

\subsection{Treatment Protocols}

2.4.1. Outpatient Rehabilitation. All participants were required to begin or to continue attending a recently arranged course of outpatient rehabilitation as prescribed by a physician and delivered at any rehabilitation clinic in the Boston area. In general, a typical course of outpatient rehabilitation (OR) for CNSLBP consists of $1 / 2$ - to 1 -hour sessions twice weekly for 4-6 weeks and may employ various combinations of analgesic and anti-inflammatory medication, joint manipulation, therapeutic exercise, cognitive behavioral treatment, and education. OR treatments were neither administered nor paid for by our study. Because of this we were not able to specify the therapeutic modalities employed, the number or frequency of treatments, or the characteristics of the therapists who delivered them. OR regimens were consequently expected to vary from one clinic to another, but there were no systematic differences between the requirements for $\mathrm{OR}$ in the two treatment groups. Participants were allowed 20 weeks to complete their course of OR. The number and frequency of treatments were determined by each participant and their therapist. Compliance with OR treatment was assessed by requesting a list of treatment dates from each participant at 20 -week follow-up.

2.4.2. Structural Integration (SI). SI aims to gradually modify chronic patterns of posture and movement to more closely approximate specific ideals that Rolf put forth as indices of optimum biomechanical efficiency, rather than to focus exclusively on local symptoms. The most important of those ideals are vertical stacking of major body segments (i.e., cranium, thorax, pelvis, and legs), left/right symmetry, horizontality of major body segments on the sagittal plane, and graceful coordination of movement for which thoracicpelvic counterrotation in gait is often taken as an index. Rolf hypothesized that the individual's ability to alter ingrained patterns of posture and movement was limited by chronic rigidities of the myofascial tissues that envelop all striated muscles. The manipulative technique of SI is believed to reduce the rigidity of these restrictions and, along with increased kinesthetic awareness, to allow the individual to more closely approximate the Rolf ideals [17].

Most often the client lies on a low, broad treatment table, but is sometimes seated or standing. The therapist uses fingers, knuckles, closed fists, or an elbow to apply sustained pressures and shearing forces of up to several minutes duration to a series of carefully selected, local areas of soft tissue. Often the patient is asked to perform specific movements as force is applied (Figure 1). SI manipulation differs from most forms of massage in the levels of force applied, which may amount to a major fraction of the practitioner's body weight. It differs from chiropractic and osteopathic manipulations in its application of force exclusively to soft tissues. Rolf regarded these features as necessary to induce remodeling of locally restricted myofasciae toward greater elasticity and mobility, but the hypothesis that such remodeling actually occurs has not been scientifically investigated. The SI therapist also teaches awareness exercises that are intended to improve the discrimination of more versus less stressful patterns of posture and movement.

As formulated by Rolf, an initial course of SI is delivered in a series of ten sessions known as the Ten Series, 
TABle 1: Rolf Ten Series treatment goals.

\begin{tabular}{ll}
\hline Session & Areas increase pliability, mobility, and L/R and A/P balance \\
\hline 1 & (i) Anterior aspect of rib cage and shoulder girdle \\
& (ii) Attachments to lateral iliac crests and greater trochanters \\
(iii) Hamstrings, iliotibial bands
\end{tabular}

L/R: left to right.

$\mathrm{A} / \mathrm{P}$ : anterior to posterior.

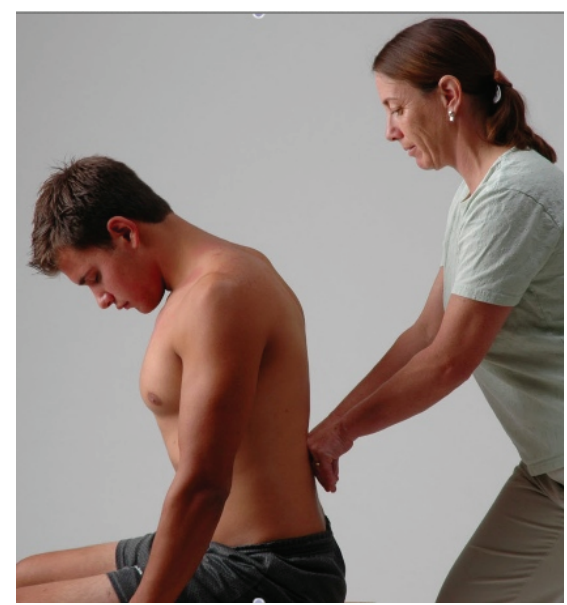

FIGURE 1: Typical SI manual technique. Courtesy Rolf Institute ${ }^{\circledR}$ of Structural Integration.

which typically includes manipulation of all major joints and anatomical segments. Each of the ten sessions is defined not by specific techniques, but rather by a set of immediate goals for biomechanical change that are intended to advance the approximation of Rolf's more overarching ideals. The aims and anatomical foci of each session are summarized in Table 1. The definition of each session in terms of goals rather than techniques allows the therapist to tailor manipulation and awareness exercises to address individual variations in posture and movement. This individualization of treatment is based on a skilled visual assessment at the beginning of each session. SI was originally provided only by therapists trained at the Rolf Institute ${ }^{\circledR}$ of Structural Integration (RISI) [23], but since the mid-1990s a number of other organizations have also provided training at widely varying levels of quality. Adequate training requires at least 300 hours.

In our study SI treatments were provided by five qualified therapists under contract. Each met the criteria of graduation from the adequate training programs of the RISI, the Guild for Structural Integration (GSI) [24], or Kinesis Myofascial Integration (KMI) [25]; a minimum of 10 years clinical practice of SI; and membership in the International Association of Structural Integrators ${ }^{\circledR}$ [26]. Two of the therapists were male and three female, ranging 10-29 years in clinical experience. One was a graduate of RISI, one was a graduate of GSI, and three were graduates of KMI. The KMI graduates agreed to provide the Rolf Ten Series instead of the twelve sessions taught by KMI, which include the Ten Series.

Ten sessions conforming to the Rolf Ten Series protocol were provided free of charge to each participant assigned to the SI + OR group. Each session lasted approximately 1 hour, and 20 weeks was allowed to receive all ten. Participants scheduled their own treatments with their choice of one of the five therapists at intervals of their own choosing. Treatments were given individually in the therapists' private practice offices and compliance was monitored from invoices that the therapists submitted to the study administrator.

In order to enhance fidelity of treatment to the Ten Series protocol, a senior SI practitioner (Eric E. Jacobson) led the therapists in a series of group discussions and reviews 
prior to the beginning of enrollment and also conducted monthly supervision sessions during the treatment phase of the study. However, there was no systematic collection of data on treatment fidelity.

2.5. Outcomes. The primary outcome was change between baseline and 20-week follow-up on a patient-rated visual analog scale $(0-100 \mathrm{~mm})$ of bothersomeness of pain on average over the preceding week with anchors at "none" $=0$ and "worst imaginable" $=100$ (VAS Pain) [27]. The secondary outcome was change in the total of the RolandMorris Disability Questionnaire (RMDQ) over the same period [28, 29]. Exploratory outcomes included the Short Form 36 Health Survey (SF36) [30], the sum of days and half days disabled over the past week, which was calculated from numeric responses to two questions adapted from Deyo et al. [31] (see items 2 and 3 of the Patient's Questionnaire; see the appendix), and Global Satisfaction with Care (GSC), a 7-point Likert scaled response to the question "Over the course of treatment for your low back pain in this study, how would you rate your overall medical care?" the last rated only at week 20 follow-up. SF36 composite scores were obtained using an online calculator, selecting the provided normative reference data "United States, 1998" [32]. These instruments are all patient-completed and have been recommended for use together in low back pain trials $[31,33]$. The Wilcoxon rank sum test was prespecified to compare change scores for each of these outcomes across treatment groups.

In addition, four psychological-cognitive variables thought to be prognostic of low back pain chronicity [34] were measured at baseline: the Hospital Anxiety and Depression Scale [35, 36], Pain Catastrophizing Scale [37], Tampa Scale of Kinesiophobia [38, 39], and Wiley-7, a scale for hypochondriasis and somatization [40].

2.5.1. Adverse Event ( $A E$ ) Monitoring. AE were monitored through reports submitted by study staff and a biweekly Patient Questionnaire (PQ) (see the appendix). The latter was drafted by the PI (Eric E. Jacobson) in response to an IRB requirement that we monitor pain ratings on a biweekly basis, because no prior instrument was available to assess AE associated with SI. This is its first application, and it has not been previously validated. The PQ includes the identical rating questions for VAS Pain and days and half days disabled as the outcome measures administered at baseline and follow-up. It also includes a list of potential treatment related experiences, both positive and negative, each of which may be endorsed as having occurred since the previous questionnaire. For each endorsed experience, an opportunity is provided to rate its duration and to enter a free text description.

AE were inferred from PQ endorsements which were a negative experience, not a symptom of low back pain, and lasting more than one hour. PQ responses were screened against these criteria by two investigators (Eric E. Jacobson, Alec L. Meleger) working independently, with differences resolved by discussion and inspection of the questionnaire responses. All endorsements on each dated PQ return that met the criteria were assumed to refer to the same AE and were attributed to the treatment group to which that participant had been assigned. AE were rated monthly and jointly by the same two investigators (Eric E. Jacobson, Alec L. Meleger) for study-relatedness (definitely, probably, possibly, or not), for seriousness (mild, moderate, or severe), and as expected or unexpected. It was not feasible to blind raters because the IRB required us to report the treatment assignment of each participant with an AE on a monthly basis. The potential for bias in ratings was mitigated by the use of objective criteria for all rating distinctions other than that between mild and moderate seriousness which was made by Alec L. Meleger on the basis of his experience as director of an outpatient rehabilitating clinic.

An IRB mandated stopping rule required us to halt the study if $>30 \%$ of enrolled participants reported VAS Pain scores $\geq 30 \mathrm{~mm}$ above baseline on two successive PQs. We recorded all such elevations as $\mathrm{AE}$, rated them as studyrelated and expected, and did not rate them for seriousness.

2.5.2. Other Feasibility Outcomes. We also collected feasibility data on the demographic characteristics of unenrolled versus enrolled candidates, compliance with treatment regimens and data collection, and retention to 20-week follow-up (Eric E. Jacobson).

2.6. Sample Size. Sample size was estimated using published data from a clinical trial of massage and a meta-analysis of trials of balneotherapy, both for low back pain [41, 42]. A sample of 36 was estimated to provide $50 \%$ power to detect a between-group difference in change in VAS Pain of $16.6 \mathrm{~mm}$ and $80 \%$ power to detect a difference of $23.8 \mathrm{~mm}$, both at the $P=0.05$ level of significance. Estimates of absolute values for minimal clinically important difference (MCID) for VAS Pain in back pain range from 15 to $19 \mathrm{~mm}$ [43-45]. That sample size was also estimated to provide more than $80 \%$ power to detect a 5-point difference in change in the RMDQ at the $P=0.05$ level. MCIDs for RMDQ have been variously estimated as absolute reductions from 2 to 5 points and as a $30 \%$ reduction from baseline [43-48]. Allowing for a 10\% dropout, a sample of 40 was chosen. This was subsequently increased to 46 in response to an unexpectedly high rate of noncompliance with the requirement to attend $\mathrm{OR}$.

2.7. Data Collection and Analysis. All data were collected by the PI (Eric E. Jacobson) at the MAL except for biweekly PQs which were collected by either mail or REDCap, a secure, web-based application designed to support data capture for research studies, that is hosted by Partners HealthCare Research Computing, Enterprise Research Infrastructure \& Services group [49]. Missing responses to items on the RMDQ and SF36 were treated according to published recommendations $[50,51]$. Because the sample size planned for each treatment group was less than 30, a nonparametric analysis of outcomes was prespecified as a Wilcoxon rank sum comparisons across groups. All analyses were intent-to-treat (ITT) with last observations carried forward. In addition, we performed a post hoc ITT responder analysis on VAS Pain 
data, following recent IMMPACT and Cochrane Back Group guidelines [52, 53]. Given the availability of biweekly VAS Pain ratings which had not been anticipated at the time our analytic plan was registered, we also performed a post hoc longitudinal analysis that combined baseline, biweekly PQ, and follow-up data using a linear mixed effects model.

Our prespecified analyses of AE were comparisons across groups of the proportions of participants with at least one study-related $\mathrm{AE}$ and the proportions with any AE studyrelated or not, using Fisher's exact tests. On an ad hoc basis we compared the number of study-related AE per participant and separately of any AE per participant using Wilcoxon rank sum. We also compared the proportions with study-related $\mathrm{AE}$ rated mild, moderate, and severe. Finally we compared proportions endorsing pain and nonpain types of AE. All data analysis was performed by Eric E. Jacobson with supervision by Roger B. Davis, using SAS 9.3 for Windows.

\section{Results}

3.1. Recruitment, Enrollment, and Participant Flow. Screening of candidates began on April 12, 2011, and enrollment was completed on March 8, 2013, for an unexpected total duration of 23 months, which included 4 months during which enrollment activities were suspended. Collection of followup data was completed on August 6, 2013. Enrolled versus unenrolled candidates were roughly equivalent demographically: enrolled candidates $(n=46)$ were $58.7 \%$ female, $78.3 \%$ white, and $6.5 \%$ Hispanic-Latino and averaged 44.3 years in age. Unenrolled candidates $(n=61)$ were $62.3 \%$ female, 81.4\% white, and 5.0\% Hispanic-Latino and averaged 43.3 years of age. The demographic and prognostic characteristics of the treatment groups were acceptably similar at baseline (Table 2). The flow of participants is displayed in Figure 2.

3.2. Compliance and Retention. Compliance with the requirement to receive OR was unexpectedly low but was not significantly different across treatment groups (Table 3). The initial treatment assignment was not altered for any participant, and we found no evidence of crossover.

Loss to 20-week follow-up was $2 / 23$ (9\%) in SI + OR and $3 / 23(13 \%)$ in OR alone, which was not significantly different. (Fisher's exact 2 -sided $P=1.000$ ) The overall rate of $11 \%$ is within the range of $10-20 \%$ loss to follow-up that has been recommended as a standard for assessing back pain trials [54].

Three of the 41 participants who provided 20-week follow-up data did so by completing the study questionnaires using the REDCap secure online facility instead of coming to the MAL. Regarding compliance with the biweekly PQ, the difference in numbers of questionnaires returned in SI + OR (median $=7$ [IQR 6, 9]) versus OR alone $(7[4,8])$ was not significantly different. (Wilcoxon rank sum 2 -sided $P=$ $0.23)$.

3.3. Prespecified Outcomes. The median reductions in VAS Pain, the primary outcome, of $-26 \mathrm{~mm}$ [IQR $-31.5,-3.0$ ] in SI + OR versus $0 \mathrm{~mm}[-24.5,6.5]$ in OR alone were not significantly different (Wilcoxon rank sum 2 -sided $P=0.075$ ) (Figure 3). However, the difference in median reductions in RMDQ, the secondary outcome, of -2 points $[-4.5,-1]$ in SI + OR versus $0[-2,0]$ in OR alone, were significantly different $(P=0.007)$ (Figure 4$)$. Two points is the smallest suggested absolute MCID for RMDQ [46].

Table 4 summarizes the outcomes. Those with $P<0.01$ include RMDQ, the SF36 subscale for Bodily Pain, and GSC, each of which had greater improvement in SI + OR. All analyses included each participant in the group to which they were allocated at randomization, $n=23$ per group.

3.4. Post Hoc Analyses. In a post hoc responder analysis we tabulated the number of participants with reductions relative to baseline that were minimal (10-20\%), moderate $(\geq 30 \%)$, and substantial $(\geq 50 \%)$ and also with absolute reductions of 20 and $40 \mathrm{~mm}$ in VAS Pain, using ITT data [52, 53]. We then compared the proportions of responders versus nonresponders at each level across groups using Fisher's exact tests. SI + OR had more responders at the minimal and moderate levels at the $P<0.05$ level of significance. Differences of all other levels were in favor of SI + OR but nonsignificant (Table 5).

In an additional post hoc analysis we constructed a linear mixed effects model of repeated measures data on VAS Pain. A total of 388 observations were available with collection times ranging from 0 to 184 days and an average time from baseline to last observation of 137 days (19.6 weeks). The final model had main effects for baseline VAS Pain, baseline RMDQ, group, days, and the days-group interaction, with random effects for intercept and days. Parameter estimates and their $P$ values from that model are given in Table 6 . The negative coefficient and small $P$ value for the days-group interaction indicate a significantly greater rate of reduction in VAS Pain for SI + OR versus OR alone. Figure 5 displays the estimated marginal means and $95 \%$ confidence bands for the days-group interaction.

3.5. Adverse Events. A total of 37 study-related AE were attributed to $15 / 22(68 \%)$ of participants in SI + OR versus $29 \mathrm{AE}$ to $14 / 23(61 \%)$ of participants in OR alone. These proportions were not significantly different across groups nor were those for participants with any $\mathrm{AE}$, study-related or not (Table 7). The numbers of AE per participant were also compared across groups using Wilcoxon rank sum, first for study-related $\operatorname{AE}(P=0.28)$ and then for all $\operatorname{AE~}(P=0.73)$, neither being significantly different.

All study-related AE were rated as mild or moderate in seriousness, none were rated as severe, and all were self-limiting; that is, none required medical treatment. The proportions of participants with mild and moderate studyrelated $\mathrm{AE}$ were not significantly different across groups (Table 7). The maximum seriousness of AE for each participant was compared across groups using Wilcoxon rank sum for study-related $(P=1.00)$ and for all AE $(P=0.84)$, neither being significant different. 
TABLE 2: Baseline characteristics of treatment groups.

\begin{tabular}{|c|c|c|c|}
\hline Variable (range) & $\mathrm{SI}+\mathrm{OR}(n=23)$ & OR alone $(n=23)$ & $P^{1}$ \\
\hline \multicolumn{4}{|l|}{ Female } \\
\hline Number (\%) & $13(56 \%)$ & $14(61 \%)$ & 0.77 \\
\hline \multicolumn{4}{|l|}{ White } \\
\hline Number (\%) & $20(87 \%)$ & $16(70 \%)$ & 0.16 \\
\hline \multicolumn{4}{|l|}{ Age years $(18-54)$} \\
\hline Mean (SD) & $43.1(13.4)$ & $45.6(14.0)$ & 0.54 \\
\hline \multicolumn{4}{|l|}{ BMI $(<40)$} \\
\hline Mean (SD) & $26.1(4.4)$ & $23.0(4.8)$ & 0.18 \\
\hline \multicolumn{4}{|c|}{ VAS Pain $(0-100 \mathrm{~mm})$} \\
\hline Mean (SD) & $46(23)$ & $50(20)$ & 0.55 \\
\hline \multicolumn{4}{|l|}{ RMDQ (0-24) } \\
\hline Mean (SD) & $7.7(4.5)$ & $7.7(5.3)$ & 1.00 \\
\hline \multicolumn{4}{|c|}{ Prescription pain medication } \\
\hline Number (\%) & $2(9 \%)$ & $5(22 \%)$ & 0.23 \\
\hline \multicolumn{4}{|l|}{ Years since onset } \\
\hline Mean (SD) & $10.7(10.9)$ & $6.6(6.3)$ & 0.12 \\
\hline \multicolumn{4}{|c|}{ Sum of days and half days disabled } \\
\hline Mean (SD) & $4.1(4.6)$ & $5.3(4.5)$ & 0.92 \\
\hline \multicolumn{4}{|c|}{ Depression (HADS-D) (0-21) } \\
\hline Mean (SD) & $3.7(3.9)$ & $3.8(3.3)$ & 0.98 \\
\hline \multicolumn{4}{|c|}{ Anxiety (HADS-A) (0-21) } \\
\hline Mean (SD) & $5.5(2.8)$ & $5.8(3.4)$ & 0.71 \\
\hline \multicolumn{4}{|c|}{ Pain catastrophizing (PCS) (0-56) } \\
\hline Mean (SD) & $14.3(11.4)$ & $15.1(10.6)$ & 0.80 \\
\hline \multicolumn{4}{|c|}{ Kinesiophobia (TSK) $(-3$ to +48$)$} \\
\hline Mean (SD) & $14.2(9.7)$ & $15.4(8.2)$ & 0.67 \\
\hline \multicolumn{4}{|c|}{ Hypochondriasis (W7-IW) (0-3) } \\
\hline Mean (SD) & $0.6(0.6)$ & $0.6(0.8)$ & 0.71 \\
\hline \multicolumn{4}{|c|}{ Somatization (W7-IC) (0-3) } \\
\hline Mean (SD) & $1.1(1.0)$ & $0.7(0.8)$ & 0.26 \\
\hline
\end{tabular}

SD: standard deviation.

BMI: body mass index.

VAS Pain: visual analogue scale of pain.

RMDQ: Roland-Morris Disability Questionnaire.

HADS-D and HADS-A: depression and anxiety subscales of the Hospital Anxiety and Depression Scale; PCS: Pain Catastrophizing Scale.

TSK: Tampa Scale of Kinesiophobia.

W7-IW and W7-IC: Illness Worry and Illness Conviction subscales of the Wiley-7.

${ }^{1} 2$-sided Student's $t$-test.

The most endorsed types of study-related AE in both groups were sharp, burning, and aching pain. The proportions of participants who endorsed the most frequent types were compared across groups, and all were nonsignificant except for a residual category of nonpain endorsements which were significantly more frequent in SI + OR $(P=0.005)$ (Table 7).

No subject reported an elevation of VAS Pain $\geq 30 \mathrm{~mm}$ above baseline on two successive PQ, and the IRB stopping rule was consequently never triggered. Two participants in $\mathrm{SI}+\mathrm{OR}$ were lost to follow-up due to AE, only one of which was study-related. The first reported an episode of "dread and worry" regarding their next treatment and subsequently dropped out, citing intolerance of the discomfort of SI treatment and a poor relationship with the SI therapist. The second dropped out subsequent to enrollment but prior to receiving any study-related treatment due to an exacerbation of a preexisting medical condition.

\section{Discussion}

This is the first randomized trial to assess the therapeutic effect of SI as an adjunct to OR for CNSLBP and only the third trial of SI for any medical condition $[55,56]$. It is the first systematic study of $\mathrm{AE}$ associated with SI treatment, which were robustly monitored by both staff and participant reports and identified and rated using conservative criteria.

Regarding feasibility outcomes, we successfully recruited and enrolled a sample whose demographic characteristics did 


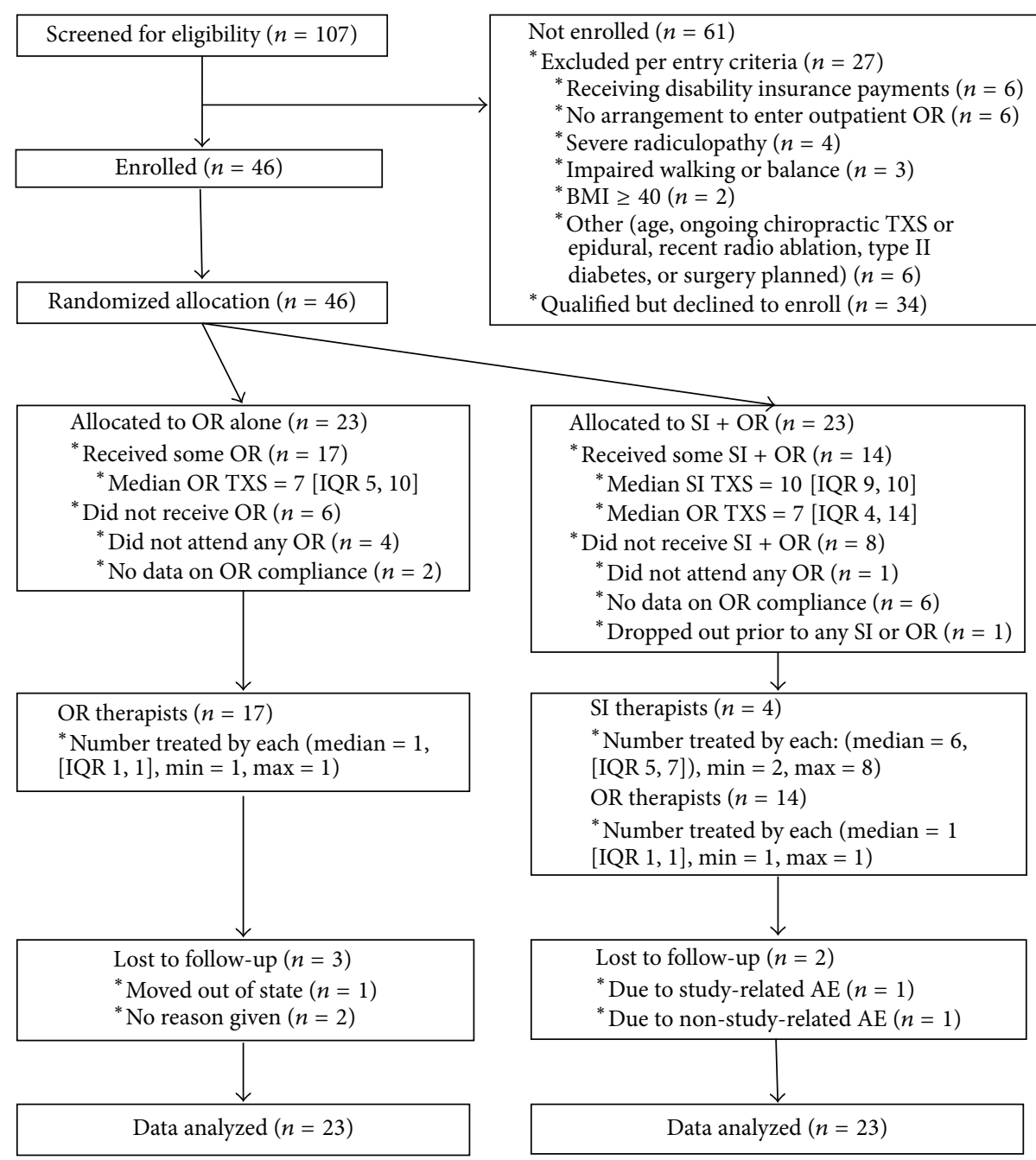

FIGURE 2: Participant flow. BMI: body mass index; IQR: interquartile range; TXS: treatments.

TABLE 3: Compliance with treatment assignments and loss to follow-up.

\begin{tabular}{|c|c|c|c|}
\hline & $\mathrm{SI}+\mathrm{OR}(n=23)$ & OR alone $(n=23)$ & $P$ \\
\hline \multicolumn{4}{|c|}{ Compliance with OR treatment } \\
\hline \multicolumn{4}{|l|}{ Number of TXS } \\
\hline Median [IQR] & $7[4,14]$ & $7[5,10]$ & $0.75^{1}$ \\
\hline \multicolumn{4}{|l|}{ PTS $\mathrm{w} / \geq 1$ treatment } \\
\hline Number (\%) & $14(61 \%)$ & $17(74 \%)$ & $0.53^{2}$ \\
\hline \multicolumn{4}{|c|}{ Compliance with SI treatment } \\
\hline \multicolumn{4}{|l|}{ Number of TXS } \\
\hline Median [IQR] & $10[9,10]$ & $0[0,0]$ & \\
\hline \multicolumn{4}{|l|}{ PTS w $/ \geq 1$ treatment } \\
\hline Number (\%) & $22(96 \%)$ & $0(0 \%)$ & \\
\hline Lost to week 20 follow-up & $2(9 \%)$ & $3(13 \%)$ & $1.000^{2}$ \\
\hline
\end{tabular}

TXS: treatments.

IQR: interquartile range.

PTS: participants.

${ }^{1}$ 2-sided Wilcoxon rank sum test; ${ }^{2} 2$-sided Fisher's exact test. 
TABLE 4: Outcomes.

\begin{tabular}{|c|c|c|c|}
\hline \multirow[t]{2}{*}{ Outcomes (range) } & \multicolumn{2}{|c|}{$\begin{array}{l}\text { Change scores } \\
\text { median [IQR] }\end{array}$} & \multirow[t]{2}{*}{$P^{1}$} \\
\hline & $\mathrm{SI}+\mathrm{OR}(n=23)$ & OR alone $(n=23)$ & \\
\hline \multicolumn{4}{|l|}{ Primary outcome } \\
\hline VAS Pain $(0-100$ mm) & $-26[-31.5,-3.0]$ & $0[-24.5,6.5]$ & 0.075 \\
\hline \multicolumn{4}{|l|}{ Secondary outcome } \\
\hline RMDQ (0-24 points) & $-2[-4.5,-1]$ & $0[-2,0]$ & $0.007^{4}$ \\
\hline \multicolumn{4}{|l|}{ Exploratory outcomes } \\
\hline Days + half days disabled $(0-14)$ & $-1.0[-3.5,0]$ & $0.0[4.5,0.5]$ & 0.445 \\
\hline \multicolumn{4}{|l|}{ SF36 subscales $(0-100)^{2}$} \\
\hline Physical function & $5[0,15]$ & $5[0,13]$ & 0.842 \\
\hline Role physical & $25[0,50]$ & $0[0,25]$ & 0.349 \\
\hline Bodily Pain & $16[7,25]$ & $0[0,11]$ & $0.009^{4}$ \\
\hline General health & $0[0,8]$ & $3[0,10]$ & 0.673 \\
\hline Vitality & $8[0,16]$ & $0[-5,5]$ & 0.034 \\
\hline Social function & $0[0,16]$ & $0[-13,0]$ & 0.041 \\
\hline Role emotional & $0[0,0]$ & $0[0,0]$ & 0.771 \\
\hline Mental health & $0[-4,8]$ & $0[-4,4]$ & 0.305 \\
\hline \multicolumn{4}{|l|}{ SF36 composite scores ${ }^{2}$} \\
\hline Physical & $3[1,10]$ & $3[0,9]$ & 0.306 \\
\hline Mental & $0[-3,3]$ & $0[-4,1]$ & 0.424 \\
\hline GSC $(\text { Likert }-3 \text { to }+3)^{3}$ & $3[2,3]$ & $2[1,2.25]$ & $0.0003^{5}$ \\
\hline
\end{tabular}

IQR: interquartile range.

VAS: Visual Analog Scale.

RMDQ: Roland-Morris Disability Scale.

SF36: Short Form (36) Health Survey.

GSC: Global Satisfaction with Care.

${ }^{1}$ Wilcoxon rank sum 2-sided; ${ }^{2}$ higher scores on SF36 subscales and composite scores indicate more positive health; ${ }^{3}$ higher scores on GSC indicate greater satisfaction with care; ${ }^{4} P<0.01 ;{ }^{5} P<0.001$.

TABle 5: VAS Pain responders.

\begin{tabular}{lcccc}
\hline Reduction & \multicolumn{3}{c}{$\begin{array}{c}\text { Responders } \\
\text { number (\%) }\end{array}$} & RR (CI) ${ }^{1}$ \\
\hline $10-20 \%$ & SI + OR $(n=23)$ & OR alone $(n=23)$ & $1.89(1.07-3.32)$ & \\
$\geq 30 \%$ & $17(74 \%)$ & $9(39 \%)$ & $2.14(1.08-4.26)$ & $0.036^{2}$ \\
$\geq 50 \%$ & $15(65 \%)$ & $7(30 \%)$ & $2.00(0.91-4.41)$ & $0.038^{2}$ \\
$\geq 20 \mathrm{~mm}$ & $12(52 \%)$ & $6(26 \%)$ & $1.71(0.83-3.56)$ & 0.130 \\
$\geq 40 \mathrm{~mm}$ & $12(52 \%)$ & $7(30 \%)$ & $2.50(0.54-11.60)$ & 0.231 \\
\hline
\end{tabular}

RR: relative risk.

CI: $95 \%$ confidence intervals.

${ }^{1}$ Fisher's exact 2 -sided; ${ }^{2} P<0.05$.

not differ significantly from those unenrolled. Randomization produced treatment groups that were acceptably equivalent on prognostically significant variables. Compliance with SI treatment was high, suggesting that any discomfort associated with it did not dissuade the majority of participants assigned to SI + OR from attending. Neither the incidence nor the seriousness of $\mathrm{AE}$ was significantly increased by the addition of SI to OR. Compliance with biweekly and follow-up data collection was acceptable and did not vary significantly between treatment groups. Loss to follow-up was within acceptable limits, and we found no evidence of crossover between treatment regimes. However, the length of time to recruit the cohort was unexpectedly long, and compliance with the requirement to receive OR treatment was unexpectedly low. Both would need to be remediated in a follow-up study.

Improvements in the primary outcome, VAS Pain, were not significantly different between treatment groups. However, improvements in the secondary outcome, RMDQ, were significantly greater in SI + OR versus OR alone, with the difference between median change scores just satisfying the lowest recommended absolute MCID [46]. Among 


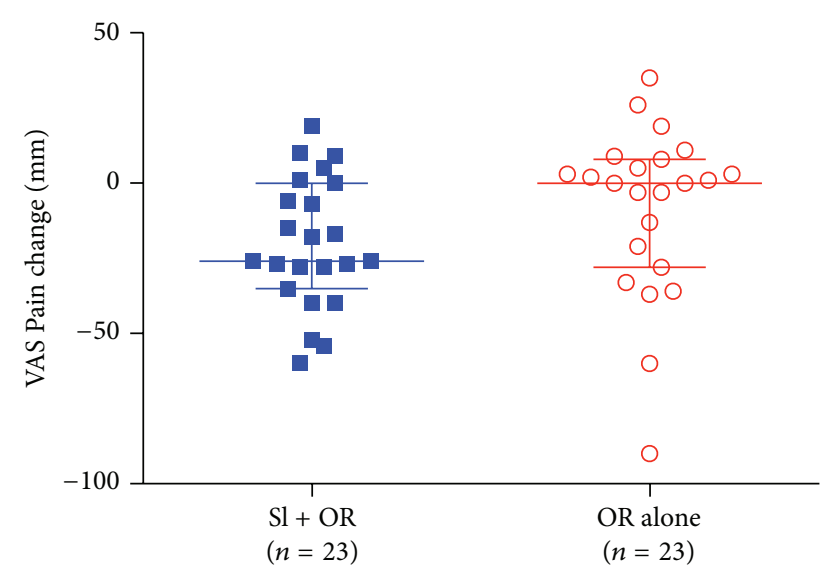

FIGURE 3: VAS Pain primary outcome. Widest horizontal lines indicate median values; narrower lines indicate interquartile ranges.

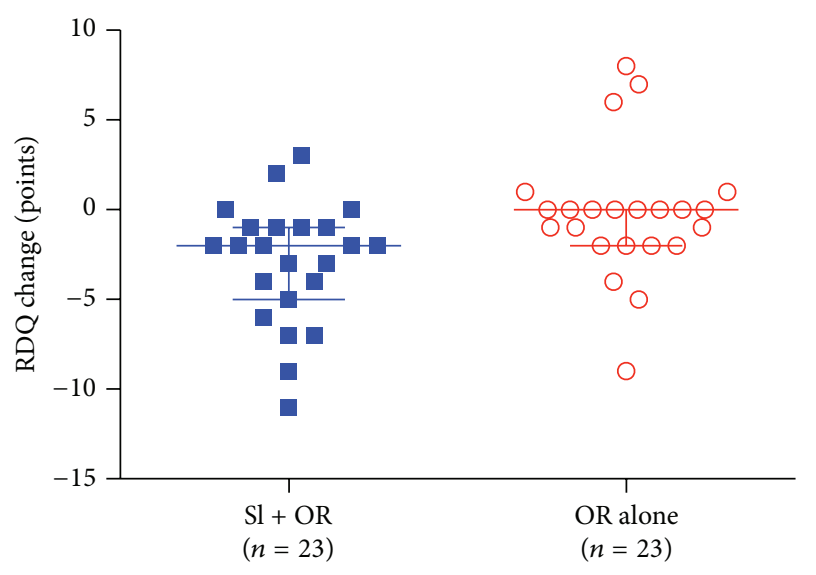

FIGURE 4: RMDQ secondary outcome. Widest horizontal lines indicate median values; narrower lines indicate interquartile ranges.

TABLE 6: Parameter estimates from linear mixed effects model of longitudinal VAS Pain data.

\begin{tabular}{lccc}
\hline Parameter & Estimate & SE & $P^{1}$ \\
\hline Intercept & $31.53 \mathrm{~mm}$ & 4.75 & $\mathrm{n} / \mathrm{a}$ \\
Group $^{2}$ & $3.06 \mathrm{~mm}$ & 4.94 & 0.5364 \\
RMDQ & $1.91 \mathrm{~mm}$ & 0.43 & $<0.0001^{3}$ \\
Days & $-0.05 \mathrm{~mm} /$ day & 0.03 & $<0.0001^{3}$ \\
Days-group $^{2}$ & $-0.14 \mathrm{~mm} /$ day & 0.05 & $0.0039^{4}$ \\
\hline
\end{tabular}

SE: standard error.

${ }^{1}$ Wald type $3 \mathrm{~F}$ tests of fixed effects; ${ }^{2}$ Group parameters estimate the amount by which the values for SI + OR differ from those for OR alone; ${ }^{3} P<0.001$; ${ }^{4} P<0.01$.

exploratory outcomes the SF36 subscale for Bodily Pain and GSC both improved more in SI + OR versus OR alone (Table 4). These outcomes suggest that the addition of SI to OR might provide additional reductions of back pain related disability and satisfaction with care at least in the short term.

In the post hoc analysis of longitudinal VAS Pain data the estimated coefficient for the group-days interaction

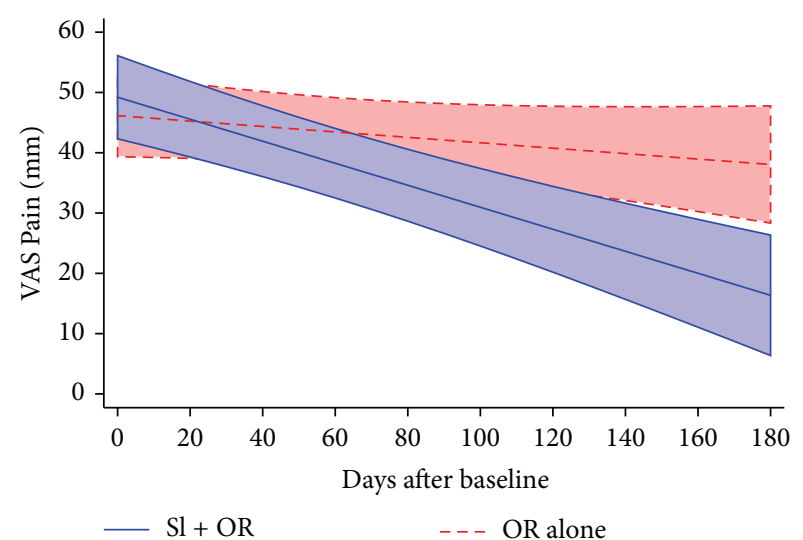

FIGURE 5: Estimated marginal means and 95\% confidence bands for days-group interaction from linear mixed effects model of VAS Pain.

$(-0.14 \mathrm{~mm} /$ day $)$, which indicates a greater rate of decline in SI + OR versus OR alone, was significant $(P=0.0039)$ (Table 6).

Because this is the only clinical trial of SI for any type of low back pain reported to date, its outcomes can only be compared to studies of other modalities of manipulation for this condition. The most relevant context might be the few other trials that have assessed the effect of manual therapies as adjuncts to outpatient rehabilitation for CNSLBP. One randomized trial for chronic low back pain included a comparison between ongoing usual care versus osteopathic manipulative therapy plus usual care. The latter produced significantly greater improvements in a VAS of pain at 1-, 3-, and 6-month follow-ups, but there were no significant differences in the RMDQ change [57]. Another randomized trial compared physician consultation alone versus consultation plus muscle energy manipulation and exercise. It found significantly greater mean reduction in VAS Pain in the latter group at both 5 and 12 months [58]. These comparisons are of limited validity because of the significant differences between SI and osteopathic or muscle energy techniques in modes of clinical assessment, treatment goals, and manipulative and educational techniques.

Comparison of our AE data with those from other studies is confounded by variation in the criteria and procedures used to identify AE associated with manual therapies [59]. A systematic review of $\mathrm{AE}$ associated with chiropractic as well as other kinds of manual therapies found that in cohort studies about $41 \%$ of patients reported minor to moderate AE following a treatment, the majority occurring within one day and resolving within two. The incidence of severe events was extremely small and none were catastrophic. The same review found that in active treatment arms of RCTs $22 \%$ of patients reported mild or moderate AE, but this was not significantly different from the rate for sham treatment [60]. However, some studies have found much lower rates. A survey of outpatients receiving $\geq 3$ treatments of osteopathic manipulation found that only $8.6 \%$ reported mild to moderate AE after treatment, most frequently pain, soreness, headache, or nausea [61]. Some RCTs of massage for LBP have also reported rates much lower than ours. 
TABLE 7: Participants with AE.

\begin{tabular}{|c|c|c|c|c|}
\hline & \multicolumn{2}{|c|}{$\begin{array}{l}\text { Participants } \\
\text { number (\%) }\end{array}$} & \multirow[t]{2}{*}{$\mathrm{RR}(\mathrm{CI})^{2}$} & \multirow[t]{2}{*}{$P^{2}$} \\
\hline & $\mathrm{SI}+\mathrm{OR}(n=22)^{1}$ & OR alone $(n=23)$ & & \\
\hline \multicolumn{5}{|l|}{ Participants with $\geq 1 \mathrm{AE}$} \\
\hline Study-related AE & $15(68 \%)^{1}$ & $14(61 \%)$ & $1.12(0.73-1.73)$ & 0.76 \\
\hline Any AE & $16(70 \%)^{3}$ & $16(70 \%)$ & $1.00(0.68-1.47)$ & 1.00 \\
\hline \multicolumn{5}{|l|}{$\begin{array}{l}\text { Participants endorsing study-related AE by } \\
\text { severity }\end{array}$} \\
\hline Mild & $12(55 \%)^{1}$ & $7(30 \%)$ & $1.79(0.87-3.70)$ & 0.14 \\
\hline Moderate & $10(45 \%)^{1}$ & $11(48 \%)$ & $0.95(0.51-1.78)$ & 1.00 \\
\hline Serious & 0 & 0 & & \\
\hline \multicolumn{5}{|l|}{$\begin{array}{l}\text { Participants endorsing types of study-related } \\
\text { AE }\end{array}$} \\
\hline Sharp, burning, aching, or other pain & $13(59 \%)^{1}$ & $9(39 \%)$ & $1.51(0.81-2.80)$ & 0.24 \\
\hline VAS Pain rating $\geq 30 \mathrm{~mm}$ above baseline & $4(18 \%)^{1}$ & $6(26 \%)$ & $0.70(0.23-2.14)$ & 0.72 \\
\hline Numbness & $3(14 \%)^{1}$ & $2(9 \%)$ & $1.57(0.29-8.51)$ & 0.67 \\
\hline Tingling & $5(23 \%)^{1}$ & $2(9 \%)$ & $2.6(0.56-12.10)$ & 0.24 \\
\hline Pulsating sensation & $2(9 \%)^{1}$ & $2(9 \%)$ & $1.05(0.16-6.78)$ & 1.00 \\
\hline $\begin{array}{l}\text { Heat, sweating, feeling dizzy or spinning, less } \\
\text { coordinated walking, less secure on feet, more } \\
\text { difficulty to move, and other nonpain events }\end{array}$ & $12(55 \%)^{1}$ & $3(13 \%)$ & $4.18(1.36-12.84)$ & $0.005^{4}$ \\
\hline
\end{tabular}

AE: adverse event; RR: risk ratio; CI: $95 \%$ confidence interval; ${ }^{1} n=22$ because one participant dropped out before receiving any treatment; ${ }^{2} \mathrm{RR}, \mathrm{CI}$, and 2 sided $P$ from Fisher's exact tests; ${ }^{3} n=23$ to include 1 drop out with a non-study-related $\mathrm{AE} ;{ }^{4} P \leq 0.01$.

One noted minor AE such as pain or discomfort in $13 \%$ of patients receiving massage [62]. Another comparing relaxation massage, structural massage, and usual care found $4 \%$ of relaxation and $7 \%$ of structural massage recipients reported $\mathrm{AE}$, mostly increased pain [63]. The higher rates found for both SI + OR (68\%) and OR alone (61\%) in our study might be due to prompting by the PQ, which invited endorsements of a list of 21 possible dysphoric sequalae to treatment, and to our conservative criterion of $>1$-hour duration for identifying $\mathrm{AE}$ from those endorsements.

4.1. Limitations. The large number of exclusion criteria, including those which excluded candidates with systemic inflammatory conditions and neurologically or pharmacologically impaired balance, might have resulted in the enrollment of a sample that was not representative of the typical clinical population, and this might limit the generalizability of our results. Obvious differences between the experiences of $\mathrm{OR}$ and SI treatment made it infeasible to blind participants or therapists to treatment allocation. Effective maintenance of the initial blinding of investigators proved to be infeasible due to limited administrative staffing. The potentially biasing effect of the latter was mitigated by the fact that all outcomes were patient-rated. Bias in the identification and rating of $\mathrm{AE}$ was mitigated by the use of objective criteria except for the distinction between mild and moderate seriousness. Statistical power to detect absolute MCIDs in the primary and secondary outcomes was limited.

Compliance with the requirement to receive OR was unexpectedly low and might have contributed to the median change scores of zero for both VAS Pain and RMDQ in the $\mathrm{OR}$ alone group. For the same reason, intent-to-treat analysis might not provide as good an estimate of the effect of adding SI to OR as would a per protocol analysis that used data only from participants who attended some number of OR treatment sessions that had been determined a priori. Similar rates of noncompliance with outpatient care for low back pain $(\sim 50 \%)$ have been reported elsewhere, but our study design also contributed to this problem in that OR was prescribed and delivered outside of our administrative purview [64]. For the same reason we were unable to monitor the specific treatment modalities utilized in OR. Our requests for data on OR attendance from participants often went unanswered. We did not directly monitor SI treatment sessions for fidelity to protocol nor require the therapists to report their treatment interventions in detail.

Because this study assessed the effect of SI as an adjunct to $\mathrm{OR}$ versus $\mathrm{OR}$ alone, its outcomes cannot be interpreted as indicative of the effect that SI alone might have on CNSLBP. The Hawthorne (time and attention) effect was likely greater in SI + OR versus OR alone because of the additional 10 hours of hands-on treatment in the former, and this might have contributed to the greater reduction in RMDQ and the slightly higher GSC scores in the SI + OR group. A placebo effect might also have contributed to outcome differentials, because members of the SI + OR group were aware that they were receiving the investigational treatment. Finally, the multiple comparisons made in our analysis of outcomes and the absence of follow-up at a longer duration are additional limitations. 
A follow-up study should provide SI according to a specific treatment protocol such as the Rolf Ten Series, should utilize therapists who are adequately trained and experienced in whatever protocol is used, and should allow for the individualization of treatment strategies to reflect actual practice. The collection of information on the specific SI techniques employed in each treatment session would enable closer monitoring of fidelity to protocol. If outpatient rehabilitation were to be a comparator, providing it within the study administration would enable better monitoring of compliance and the collection of data on the specific treatment modalities used. At least a three-month follow-up should be included. We speculate that SI alone might be superior to outpatient rehabilitation alone and note that a direct comparison of the two could control for time, attention, and cutaneous stimulation across treatment groups.

Our positive outcome for greater reduction in disability in the SI + OR group suggests that hypothesized mechanisms for mediating a therapeutic effect of SI are also worthy of future investigation.

\section{Conclusions}

Data on enrollment, retention, data collection, and compliance with SI treatment suggest that a follow-up study would be feasible. However, the study design would have to increase the efficiency of recruitment and improve compliance with OR. The outcomes suggest that adding SI to outpatient rehabilitation for CNSLBP is not likely to enhance reductions in patient-rated pain but is likely to enhance reductions in low back pain related disability at least for the short term and to modestly increase patient satisfaction without significantly increasing the rates or seriousness of $\mathrm{AE}$. If these indications were confirmed by a more definitive study, that might support the recommendation of SI as an effective adjunct to outpatient rehabilitation for CNSLBP.

\section{Appendix}

\section{A. Patient's Questionnaire}

Please complete the following questions privately so that your answers are not influenced by anyone else.

Thank you!

Please enter the date you completed this questionnaire:

$$
- \text { (yyyy-mm-dd) }
$$

A.1. How Bothersome Has Your Low Back Pain Been over the Past Week? Move the slider along the line to the place between "not at all" and "worst imaginable" that shows your answer.

$$
0=\text { not at all } \quad \begin{aligned}
& 100=\text { worst } \\
& \text { imaginable }
\end{aligned}
$$

(Place a mark on the scale above)
A.2. Please Answer the following Questions by Selecting the Answer That Is Right for You from the Drop Down Menus

During the past week, how many days was your activity restricted due to low back problems?

$$
\begin{aligned}
& \square 0 \\
& \square 1 \\
& \square 2 \\
& \square 3 \\
& \square 4 \\
& \square 5 \\
& \square 6 \\
& \square 7 \\
& \text { (select from menu) }
\end{aligned}
$$

During the past week, how many half-days did you spend either in bed, home from work or school, or cutting down on usual activities because of low back pain?

$$
\begin{aligned}
& \square \text { no half days } \\
& \square \text { one half day } \\
& \square \text { two half days } \\
& \square \text { three half days } \\
& \square \text { four half days } \\
& \square \text { five half days } \\
& \square \text { six half days } \\
& \square \text { seven half days } \\
& \square \text { eight half days } \\
& \square \text { nine half days } \\
& \square \text { ten half days } \\
& \square \text { eleven half days } \\
& \square \text { twelve half days } \\
& \square \text { thirteen half days } \\
& \square \text { fourteen half days } \\
& \text { (select from drop down menu) }
\end{aligned}
$$

A.3. If You Have Had Any of the Experiences Listed below during or following Your Most Recent Treatment, Please Check the Box Next to It. Only Check Experiences That You Definitely Had

$\square(1)$ Numbness

Duration during treatment

$$
\begin{aligned}
& \square \text { a few minutes } \\
& \square \text { more than a few minutes } \\
& \square \text { most of the session }
\end{aligned}
$$

Duration after treatment

$$
\begin{aligned}
& \square \text { a few minutes } \\
& \square \text { more than a few minutes up to an hour } \\
& \square \text { more than one hour } \\
& \square \text { about one day } \\
& \square \text { several days } \\
& \square \text { one week or more }
\end{aligned}
$$

Please tell us more - 
$\square$ (2) Tingling

Duration during treatment

$$
\begin{aligned}
& \square \text { a few minutes } \\
& \square \text { more than a few minutes } \\
& \square \text { most of the session }
\end{aligned}
$$

Duration after treatment

$$
\begin{aligned}
& \square \text { a few minutes } \\
& \square \text { more than a few minu } \\
& \square \text { more than one hour } \\
& \square \text { about one day } \\
& \square \text { several days } \\
& \square \text { one week or more }
\end{aligned}
$$$$
\square \text { more than a few minutes up to an hour }
$$

Please tell us more -

$\square(3)$ Heat

Duration during treatment

$$
\begin{aligned}
& \square \text { a few minutes } \\
& \square \text { more than a few minutes } \\
& \square \text { most of the session }
\end{aligned}
$$

Duration after treatment

$$
\begin{aligned}
& \square \text { a few minutes } \\
& \square \text { more than a few min } \\
& \square \text { more than one hour } \\
& \square \text { about one day } \\
& \square \text { several days } \\
& \square \text { one week or more }
\end{aligned}
$$$$
\square \text { more than a few minutes up to an hour }
$$

Please tell us more -

$\square(4)$ Cold

Duration during treatment

$$
\begin{aligned}
& \square \text { a few minutes } \\
& \square \text { more than a few minutes } \\
& \square \text { most of the session }
\end{aligned}
$$

Duration after treatment

$$
\begin{aligned}
& \square \text { a few minutes } \\
& \square \text { more than a few minutes up to an hour } \\
& \square \text { more than one hour } \\
& \square \text { about one day } \\
& \square \text { several days } \\
& \square \text { one week or more }
\end{aligned}
$$

Please tell us more -

\section{$\square$ (5) Sweating}

Duration during treatment

$$
\begin{aligned}
& \square \text { a few minutes } \\
& \square \text { more than a few minutes } \\
& \square \text { most of the session }
\end{aligned}
$$

Duration after treatment

$$
\begin{aligned}
& \square \text { a few minutes } \\
& \square \text { more than a few minutes up to an hour } \\
& \square \text { more than one hour } \\
& \square \text { about one day }
\end{aligned}
$$

$$
\begin{aligned}
& \square \text { several days } \\
& \square \text { one week or more }
\end{aligned}
$$

Please tell us more -

$\square(6)$ Breathing fast

Duration during treatment

$$
\begin{aligned}
& \square \text { a few minutes } \\
& \square \text { more than a few minutes } \\
& \square \text { most of the session }
\end{aligned}
$$

Duration after treatment

$$
\begin{aligned}
& \square \text { a few minutes } \\
& \square \text { more than a few minutes up to an hour } \\
& \square \text { more than one hour } \\
& \square \text { about one day } \\
& \square \text { several days } \\
& \square \text { one week or more }
\end{aligned}
$$

Please tell us more -

$\square$ (7) Breathing very slow or stopped for a moment

Duration during treatment

$$
\begin{aligned}
& \square \text { a few minutes } \\
& \square \text { more than a few minutes } \\
& \square \text { most of the session }
\end{aligned}
$$

Duration after treatment

$$
\begin{aligned}
& \square \text { a few minutes } \\
& \square \text { more than a few min } \\
& \square \text { more than one hour } \\
& \square \text { about one day } \\
& \square \text { several days } \\
& \square \text { one week or more }
\end{aligned}
$$$$
\square \text { more than a few minutes up to an hour }
$$

Please tell us more -

$\square$ (8) Feeling dizzy or a spinning sensation

Duration during treatment

$$
\begin{aligned}
& \square \text { a few minutes } \\
& \square \text { more than a few minutes } \\
& \square \text { most of the session }
\end{aligned}
$$

Duration after treatment

$$
\begin{aligned}
& \square \text { a few minutes } \\
& \square \text { more than a few min } \\
& \square \text { more than one hour } \\
& \square \text { about one day } \\
& \square \text { several days } \\
& \square \text { one week or more }
\end{aligned}
$$$$
\square \text { more than a few minutes up to an hour }
$$

Please tell us more -

$\square(9)$ Feeling an emotion (please write in the name)

Duration during treatment

$$
\begin{aligned}
& \square \text { a few minutes } \\
& \square \text { more than a few minutes } \\
& \square \text { most of the time }
\end{aligned}
$$


Duration after treatment

$$
\begin{aligned}
& \square \text { a few minutes } \\
& \square \text { more than a few minutes up to an hour } \\
& \square \text { more than one hour } \\
& \square \text { about one day } \\
& \square \text { several days } \\
& \square \text { one week or more }
\end{aligned}
$$

Please tell us more -

$\square$ (10) Trembling or shaking

Duration during treatment

$$
\begin{aligned}
& \square \text { a few minutes } \\
& \square \text { more than a few minutes } \\
& \square \text { most of the session }
\end{aligned}
$$

Duration after treatment

$$
\begin{aligned}
& \square \text { a few minutes } \\
& \square \text { more than a few minutes up to an hour } \\
& \square \text { more than one hour } \\
& \square \text { about one day } \\
& \square \text { several days } \\
& \square \text { one week or more }
\end{aligned}
$$

Please tell us more -

$\square$ (11) Remembering something from your past

Duration during treatment

$$
\begin{aligned}
& \square \text { a few minutes } \\
& \square \text { more than a few minutes } \\
& \square \text { most of the session }
\end{aligned}
$$

Duration after treatment

$$
\begin{aligned}
& \square \text { a few minutes } \\
& \square \text { more than a few minutes up to an hour } \\
& \square \text { more than one hour } \\
& \square \text { about one day } \\
& \square \text { several days } \\
& \square \text { one week or more }
\end{aligned}
$$

Please tell us more -

\section{$\square(12)$ Nausea}

Duration during treatment

$$
\begin{aligned}
& \square \text { a few minutes } \\
& \square \text { more than a few minutes } \\
& \square \text { most of the session }
\end{aligned}
$$

Duration after treatment

$$
\begin{aligned}
& \square \text { a few minutes } \\
& \square \text { more than a few minutes up to an hour } \\
& \square \text { more than one hour } \\
& \square \text { about one day } \\
& \square \text { several days } \\
& \square \text { one week or more }
\end{aligned}
$$

Please tell us more - $\square(13)$ Feeling very relaxed

Duration during treatment

$$
\begin{aligned}
& \square \text { a few minutes } \\
& \square \text { more than a few minutes } \\
& \square \text { most of the session }
\end{aligned}
$$

Duration after treatment

$$
\begin{aligned}
& \square \text { a few minutes } \\
& \square \text { more than a few minutes up to an hour } \\
& \square \text { more than one hour } \\
& \square \text { about one day } \\
& \square \text { several days } \\
& \square \text { one week or more }
\end{aligned}
$$

Please tell us more -

$\square$ (14) Feeling that part of your body was pulsating

Duration during treatment

$$
\begin{aligned}
& \square \text { a few minutes } \\
& \square \text { more than a few minutes } \\
& \square \text { most of the session }
\end{aligned}
$$

Duration after treatment

$$
\begin{aligned}
& \square \text { a few minutes } \\
& \square \text { more than a few min } \\
& \square \text { more than one hour } \\
& \square \text { about one day } \\
& \square \text { several days } \\
& \square \text { one week or more }
\end{aligned}
$$$$
\square \text { more than a few minutes up to an hour }
$$

Please tell us more -

$\square$ (15) Feeling pleasantly warm

\section{Duration during treatment}

$$
\begin{aligned}
& \square \text { a few minutes } \\
& \square \text { more than a few minutes } \\
& \square \text { most of the session }
\end{aligned}
$$

Duration after treatment

$$
\begin{aligned}
& \square \text { a few minutes } \\
& \square \text { more than a few min } \\
& \square \text { more than one hour } \\
& \square \text { about one day } \\
& \square \text { several days } \\
& \square \text { one week or more }
\end{aligned}
$$$$
\square \text { more than a few minutes up to an hour }
$$

Please tell us more -

$\square$ (16) Feeling much lighter or weightless

Duration during treatment

$$
\begin{aligned}
& \square \text { a few minutes } \\
& \square \text { more than a few minutes } \\
& \square \text { most of the session }
\end{aligned}
$$

\section{Duration after treatment}

$$
\begin{aligned}
& \square \text { a few minutes } \\
& \square \text { more than a few minutes up to an hour }
\end{aligned}
$$


$\square$ more than one hour

$\square$ about one day

$\square$ several days

$\square$ one week or more

Please tell us more -

$\square$ (17) Feeling heavier

Duration during treatment

$\square$ a few minutes

$\square$ more than a few minutes

$\square$ most of the session

Duration after treatment

$\square$ a few minutes

$\square$ more than a few minutes up to an hour

$\square$ more than one hour

$\square$ about one day

$\square$ several days

$\square$ one week or more

Please tell us more -

$\square$ (18) Feeling more coordinated as you walk

Duration during treatment

$\square$ a few minutes

$\square$ more than a few minutes

$\square$ most of the session

Duration after treatment

$\square$ a few minutes
$\square$ more than a few minutes up to an hour
$\square$ more than one hour
$\square$ about one day
$\square$ several days
$\square$ one week or more

Please tell us more -

$\square$ (19) Feeling less coordinated as you walk

Duration during treatment

$\square$ a few minutes

$\square$ more than a few minutes

$\square$ most of the session

Duration after treatment
$\square$ a few minutes
$\square$ more than a few minutes up to an hour
$\square$ more than one hour
$\square$ about one day
$\square$ several days
$\square$ one week or more

Please tell us more -

$\square(20)$ Feeling more secure on your feet

Duration during treatment

$$
\begin{aligned}
& \square \text { a few minutes } \\
& \square \text { more than a few minutes } \\
& \square \text { most of the session }
\end{aligned}
$$

Duration after treatment

$\square$ a few minutes

$\square$ more than a few minutes up to an hour

$\square$ more than one hour

$\square$ about one day

$\square$ several days

$\square$ one week or more

Please tell us more -

$\square$ (21) Feeling less secure on your feet

Duration during treatment

$$
\begin{aligned}
& \square \text { a few minutes } \\
& \square \text { more than a few minutes } \\
& \square \text { most of the session }
\end{aligned}
$$

Duration after treatment

$$
\begin{aligned}
& \square \text { a few minutes } \\
& \square \text { more than a few minutes up to an hour } \\
& \square \text { more than one hour } \\
& \square \text { about one day } \\
& \square \text { several days } \\
& \square \text { one week or more }
\end{aligned}
$$

Please tell us more -

$\square$ (22) Easier to breathe

Duration during treatment

$$
\begin{aligned}
& \square \text { a few minutes } \\
& \square \text { more than a few minutes } \\
& \square \text { most of the session }
\end{aligned}
$$

Duration after treatment

$$
\begin{aligned}
& \square \text { a few minutes } \\
& \square \text { more than a few minutes up to an hour } \\
& \square \text { more than one hour } \\
& \square \text { about one day } \\
& \square \text { several days } \\
& \square \text { one week or more }
\end{aligned}
$$

Please tell us more -

$\square$ (23) More difficult to breathe

Duration during treatment

$$
\begin{aligned}
& \square \text { a few minutes } \\
& \square \text { more than a few minutes } \\
& \square \text { most of the session }
\end{aligned}
$$

Duration after treatment

$$
\begin{aligned}
& \square \text { a few minutes } \\
& \square \text { more than a few minutes up to an hour } \\
& \square \text { more than one hour } \\
& \square \text { about one day } \\
& \square \text { several days } \\
& \square \text { one week or more }
\end{aligned}
$$

Please tell us more - 
$\square$ (24) Easier to move

Duration during treatment

$$
\begin{aligned}
& \square \text { a few minutes } \\
& \square \text { more than a few minutes } \\
& \square \text { most of the session }
\end{aligned}
$$

Duration after treatment

$$
\begin{aligned}
& \square \text { a few minutes } \\
& \square \text { more than a few minutes up to an hour } \\
& \square \text { more than one hour } \\
& \square \text { about one day } \\
& \square \text { several days } \\
& \square \text { one week or more }
\end{aligned}
$$

Please tell us more -

$\square$ (25) More difficult to move

Duration during treatment

$$
\begin{aligned}
& \square \text { a few minutes } \\
& \square \text { more than a few minutes } \\
& \square \text { most of the session }
\end{aligned}
$$

Duration after treatment

$$
\begin{aligned}
& \square \text { a few minutes } \\
& \square \text { more than a few minutes up to an hour } \\
& \square \text { more than one hour } \\
& \square \text { about one day } \\
& \square \text { several days } \\
& \square \text { one week or more }
\end{aligned}
$$

Please tell us more -

$\square$ (26) Feeling like you were outside your body.

Duration during treatment

$$
\begin{aligned}
& \square \text { a few minutes } \\
& \square \text { more than a few minutes } \\
& \square \text { most of the session }
\end{aligned}
$$

Duration after treatment

$$
\begin{aligned}
& \square \text { a few minutes } \\
& \square \text { more than a few min } \\
& \square \text { more than one hour } \\
& \square \text { about one day } \\
& \square \text { several days } \\
& \square \text { one week or more }
\end{aligned}
$$$$
\square \text { more than a few minutes up to an hour }
$$

Please tell us more -

$\square$ (27) Feeling like you were more inside your body.

Duration during treatment

$$
\begin{aligned}
& \square \text { a few minutes } \\
& \square \text { more than a few minutes } \\
& \square \text { most of the session }
\end{aligned}
$$

Duration after treatment

$$
\begin{aligned}
& \square \text { a few minutes } \\
& \square \text { more than a few minutes up to an hour }
\end{aligned}
$$

$$
\begin{aligned}
& \square \text { more than one hour } \\
& \square \text { about one day } \\
& \square \text { several days } \\
& \square \text { one week or more }
\end{aligned}
$$

Please tell us more -

$\square$ (28) Sharp pain

Duration during treatment

$$
\begin{aligned}
& \square \text { a few minutes } \\
& \square \text { more than a few minutes } \\
& \square \text { most of the session }
\end{aligned}
$$

Duration after treatment

$$
\begin{aligned}
& \square \text { a few minutes } \\
& \square \text { more than a few minutes up to an hour } \\
& \square \text { more than one hour } \\
& \square \text { about one day } \\
& \square \text { several days } \\
& \square \text { one week or more }
\end{aligned}
$$

Please tell us more -

$\square$ (29) Burning pain

Duration during treatment

$$
\begin{aligned}
& \square \text { a few minutes at most } \\
& \square \text { more than a few minutes } \\
& \square \text { most of the session }
\end{aligned}
$$

Duration after treatment

$$
\begin{aligned}
& \square \text { a few minutes } \\
& \square \text { more than a few minutes up to an hour } \\
& \square \text { more than one hour } \\
& \square \text { about one day } \\
& \square \text { several days } \\
& \square \text { one week or more }
\end{aligned}
$$

Please tell us more -

$\square$ (30) Aching pain

Duration during the treatment

$$
\begin{aligned}
& \square \text { a few minutes at most } \\
& \square \text { more than a few minutes } \\
& \square \text { most of the session }
\end{aligned}
$$

Duration after treatment

$$
\begin{aligned}
& \square \text { a few minutes } \\
& \square \text { more than a few minutes up to an hour } \\
& \square \text { more than one hour } \\
& \square \text { about one day } \\
& \square \text { several days } \\
& \square \text { one week or more }
\end{aligned}
$$

Please tell us more -

A.4. Other Experiences. Please describe any other unusual positive or negative experiences in the space below. Also tell us when they started and how long they lasted. 


\section{Disclaimer}

The supporting organizations had no role in drafting or editing the paper, which are the sole responsibility of the authors.

\section{Conflict of Interests}

The primary author (Eric E. Jacobson) is a member of the Research Committee of the Rolf Institute ${ }^{\circledR}$ of Structural Integration, the Scientific Advisory Panel of the Ida P. Rolf Research Foundation, a principal in Structural Integration Trainings, LLC, and maintains a private practice in Structural Integration. The other authors have no competing interests to declare.

\section{Acknowledgments}

This study and Dr. Jacobson's effort were funded by a career development award from the National Center for Complementary and Alternative Medicine, National Institutes of Health (NCCAM/NIH, K01AT004916). Supplemental funding was provided by the Ida P. Rolf Research Foundation, Harvard Medical School, the Rolf Institute ${ }^{\circledR}$ of Structural Integration, Dean Rollings, and the Tikkun Endowment. Dr. Kaptchuk's effort was supported by a mentorship award from NCCAM/NIH (K24AT004095). Administrative and technical support was generously provided by the Motion Analysis Laboratory, Spaulding Rehabilitation Hospital, Partners Healthcare, LLC. We also thank the volunteers who served as independent monitor and on the data safety monitoring committee and the SI practitioners (Lou Benson, Lisa Grey, Ellen Halpern, Tim Roode, and Garret Whitney).

\section{References}

[1] G. B. J. Andersson, "Epidemiological features of chronic lowback pain," The Lancet, vol. 354, no. 9178, pp. 581-585, 1999.

[2] T. Vos, A. D. Flaxman, M. Naghavi et al., "Years lived with disability (YLDs) for 1160 sequelae of 289 disease and injuries 1990-2010: a systematic analysis for the Global Burden of Disease Study 2010," The Lancet, vol. 380, pp. 2163-2196, 2013.

[3] X. Luo, R. Pietrobon, S. X. Sun, G. G. Liu, and L. Hey, "Estimates and patterns of direct health care expenditures among individuals with back pain in the United States," Spine, vol. 29, no. 1, pp. 79-86, 2004.

[4] M. von Korff and K. Saunders, "The course of back pain in primary care," Spine, vol. 21, no. 24, pp. 2833-2837, 1996.

[5] R. A. Deyo and J. N. Weinstein, "Low back pain," The New England Journal of Medicine, vol. 344, no. 5, pp. 363-370, 2001.

[6] S. Dagenais, A. C. Tricco, and S. Haldeman, "Synthesis of recommendations for the assessment and management of low back pain from recent clinical practice guidelines," Spine, vol. 10, no. 6, pp. 514-529, 2010.

[7] T. J. Lee, "Pharmacologic treatment for low back pain: one component of pain care," Physical Medicine \& Rehabilitation Clinics of North America, vol. 21, no. 4, pp. 793-800, 2010.

[8] T. Kuijpers, M. van Middelkoop, S. M. Rubinstein et al., "A systematic review on the effectiveness of pharmacological interventions for chronic non-specific low-back pain," European Spine Journal, vol. 20, no. 1, pp. 40-50, 2011.
[9] A. Stout, "Epidural steroid injections for low back pain," Physical Medicine and Rehabilitation Clinics of North America, vol. 21, no. 4, pp. 825-834, 2010.

[10] M. W. van Tulder, B. Koes, and A. Malmivaara, "Outcome of non-invasive treatment modalities on back pain: an evidencebased review," European Spine Journal, vol. 15, supplement 1, pp. S64-S81, 2006.

[11] T. Kuijpers, M. van Middelkoop, S. M. Rubinstein et al., "A systematic review on the effectiveness of pharmacological interventions for chronic non-specific low-back pain," European Spine Journal, vol. 20, no. 1, pp. 40-50, 2011.

[12] P. M. Wolsko, D. M. Eisenberg, R. B. Davis, R. Kessler, and R. S. Phillips, "Patterns and perceptions of care for treatment of back and neck pain: results of a national survey," Spine, vol. 28, no. 3, pp. 292-297, 2003.

[13] D. C. Cherkin, R. A. Deyo, K. J. Sherman et al., "Characteristics of visits to licensed acupuncturists, chiropractors, massage therapists, and naturopathic physicians," Journal of the American Board of Family Practice, vol. 15, no. 6, pp. 463-472, 2002.

[14] J. T. Cottingham and J. Maitland, "A three-paradigm treatment model using soft tissue mobilization and guided movementawareness techniques for a patient with chronic low back pain: a case study," Journal of Orthopaedic \& Sports Physical Therapy, vol. 26, no. 3, pp. 155-167, 1997.

[15] J. E. Deutsch, L. L. Derr, P. Judd, and B. Reuven, "Treatment of chronic pain through the use of structural integration (Rolfing)," Orthopaedic Physical Therapy Clinics of North America, vol. 9, no. 3, pp. 411-425, 2000.

[16] H. James, L. Castaneda, M. E. Miller, and T. Findley, "Rolfing structural integration treatment of cervical spine dysfunction," Journal of Bodywork and Movement Therapies, vol. 13, no. 3, pp. 229-238, 2009.

[17] E. Jacobson, "Structural integration, an alternative method of manual therapy and sensorimotor education," The Journal of Alternative and Complementary Medicine, vol. 17, no. 10, pp. 891899, 2011.

[18] A. Considine, "Rolfing, excruciatingly helpful," New York Times, 2010.

[19] R. Krotenberg, "Alternative therapies join the mainstream," in Focus on Rehabilitation, p. 5, Kessler Institute for Rehabilitation, West Orange, NJ, USA, 2004.

[20] H. D. Kerr, "Ureteral stent displacement associated with deep massage," Western Medical Journal, vol. 96, no. 12, pp. 57-58, 1997.

[21] J. Perry, M. H. Jones, and L. Thomas, "Functional evaluation of Rolfing in cerebral palsy," Developmental Medicine and Child Neurology, vol. 23, no. 6, pp. 717-729, 1981.

[22] Partners HealthCare System, RSVP for Health: Partner with Us as a Study Volunteer!, Partners HealthCare, LLC, 2003, http:// rsvpforhealth.org/.

[23] Rolf Institute ${ }^{\circledR}$ of Structural Integration, Rolf Institute ${ }^{\circledR}$ of Structural Integration, 2014, http://www.rolf.org/.

[24] The Guild for Structural Integration, 2014, http://www.rolfguild .org.

[25] Kinesis Myofascial Integration, 2012, http://www.anatomytrains.com $/ \mathrm{kmi}$.

[26] International Association of Structural Integrators ${ }^{\circledR}, 2014$, http://www.theiasi.net/home-header.

[27] K. M. Dunn and P. R. Croft, "Classification of low back pain in primary care: using "bothersomeness" to identify the most severe cases," Spine, vol. 30, no. 16, pp. 1887-1892, 2005. 
[28] M. Roland and R. Morris, "A study of the natural history of back pain. Part I: development of a reliable and sensitive measure of disability in low-back pain," Spine, vol. 8, no. 2, pp. 141-144, 1983.

[29] M. Roland and J. Fairbank, "The Roland-Morris disability questionnaire and the Oswestry disability questionnaire," Spine, vol. 25 , no. 24, pp. 3115-3124, 2000.

[30] J. E. Ware Jr. and C. D. Sherbourne, "The MOS 36-item shortform health survey (SF-36). I. Conceptual framework and item selection," Medical Care, vol. 30, no. 6, pp. 473-483, 1992.

[31] R. A. Deyo, M. Battie, A. J. H. M. Beurskens et al., "Outcome measures for low back pain research: a proposal for standardized use," Spine, vol. 23, no. 18, pp. 2003-2013, 1998.

[32] QualityMetric, SF-36 PCS, MCS and NBS Calculator, QualityMetric, Inc., 2014, http://www.sf-36.org/nbscalc/index.shtml.

[33] C. Bombardier, "Outcome assessments in the evaluation of treatment of spinal disorders: summary and general recommendations," Spine, vol. 25, no. 24, pp. 3100-3103, 2000.

[34] I. E. J. Swinkels-Meewisse, J. Roelofs, R. A. B. Oostendorp, A. L. M. Verbeek, and J. W. S. Vlaeyen, "Acute low back pain: pain-related fear and pain catastrophizing influence physical performance and perceived disability," Pain, vol. 120, no. 1-2, pp. 36-43, 2006.

[35] A. S. Zigmond and R. P. Snaith, "The hospital anxiety and depression scale," Acta Psychiatrica Scandinavica, vol. 67, no. 6, pp. 361-370, 1983.

[36] R. P. Snaith and A. Zigmond, "The hospital anxiety and depression scale," British Medical Journal (Clinical Research Edition), vol. 292, p. 344, 1986.

[37] M. J. L. Sullivan, S. R. Bishop, and J. Pivik, "The pain catastrophizing scale: development and validation,” Psychological Assessment, vol. 7, no. 4, pp. 524-532, 1995.

[38] D. J. French, C. R. France, F. Vigneau, J. A. French, and R. T. Evans, "Fear of movement/(re)injury in chronic pain: a psychometric assessment of the original English version of the Tampa scale for kinesiophobia (TSK)," Pain, vol. 127, no. 1-2, pp. 42-51, 2007.

[39] E. J. C. M. Swinkels-Meewisse, R. A. H. M. Swinkels, A. L. M. Verbeek, J. W. S. Vlaeyen, and R. A. B. Oostendorp, "Psychometric properties of the Tampa Scale for kinesiophobia and the fear-avoidance beliefs questionnaire in acute low back pain," Manual Therapy, vol. 8, no. 1, pp. 29-36, 2003.

[40] P. Fink, H. Ewald, J. Jensen et al., "Screening for somatization and hypochondriasis in primary care and neurological inpatients: a seven-item scale for hypochondriasis and somatization," Journal of Psychosomatic Research, vol. 46, no. 3, pp. 261273, 1999.

[41] D. C. Cherkin, D. Eisenberg, K. J. Sherman et al., "Randomized trial comparing traditional Chinese medical acupuncture, therapeutic massage, and self-care education for chronic low back pain," Archives of Internal Medicine, vol. 161, no. 8, pp. 1081-1088, 2001.

[42] M. H. Pittler, M. Z. Karagülle, M. Karagulle, and E. Ernst, "Spa therapy and balneotherapy for treating low back pain: metaanalysis of randomized trials," Rheumatology (Oxford), vol. 45, no. 7, pp. 880-884, 2006.

[43] D. L. Patrick, R. Q. Deyo, S. J. Atlas et al., "Assessing healthrelated quality of life in patients with sciatica," Spine, vol. 20, no. 17, pp. 1899-1909, 1995.

[44] O. Hägg, P. Fritzell, and A. Nordwall, "The clinical importance of changes in outcome scores after treatment for chronic low back pain," European Spine Journal, vol. 12, no. 1, pp. 12-20, 2003.
[45] R. W. J. G. Ostelo, R. A. Deyo, P. Stratford et al., "Interpreting change scores for pain and functional status in low back pain: towards international consensus regarding minimal important change," Spine, vol. 33, no. 1, pp. 90-94, 2008.

[46] C. Bombardier, J. Hayden, and D. E. Beaton, "Minimal clinically important difference. Low back pain: outcome measures," The Journal of Rheumatology, vol. 28, no. 2, pp. 431-438, 2001.

[47] E. F. Maughan and J. S. Lewis, "Outcome measures in chronic low back pain," European Spine Journal, vol. 19, no. 9, pp. 14841494, 2010.

[48] K. Jordan, K. M. Dunn, M. Lewis, and P. Croft, "A minimal clinically important difference was derived for the RolandMorris Disability Questionnaire for low back pain," Journal of Clinical Epidemiology, vol. 59, no. 1, pp. 45-52, 2006.

[49] P. A. Harris, R. Taylor, R. Thielke, J. Payne, N. Gonzalez, and J. G. Conde, "Research electronic data capture (REDCap)a metadata-driven methodology and workflow process for providing translational research informatics support," Journal of Biomedical Informatics, vol. 42, no. 2, pp. 377-381, 2009.

[50] P. Kent and H. H. Lauridsen, "Managing missing scores on the Roland Morris disability questionnaire," Spine, vol. 36, no. 22, pp. 1878-1884, 2011.

[51] J. E. Ware, “SF-36 health survey update," Spine, vol. 25, no. 24, pp. 3130-3139, 2000.

[52] R. H. Dworkin, D. C. Turk, K. W. Wyrwich et al., "Interpreting the clinical importance of treatment outcomes in chronic pain clinical trials: IMMPACT recommendations," Journal of Pain, vol. 9, no. 2, pp. 105-121, 2008.

[53] A. D. Furlan, V. Pennick, C. Bombardier, and M. van Tulder, "2009 Updated method guidelines for systematic reviews in the Cochrane Back Review Group," Spine, vol. 34, no. 18, pp. 19291941, 2009.

[54] B. W. Koes, L. M. Bouter, and G. J. M. G. van der Heijden, "Methodological quality of randomized clinical trials on treatment efficacy in low back pain," Spine, vol. 20, no. 2, pp. 228-235, 1995.

[55] A. B. Hansen, K. S. Price, and H. M. Feldman, "Myofascial structural integration: a promising complementary therapy for young children with spastic cerebral palsy," Journal of EvidenceBased Complementary and Alternative Medicine, vol. 17, no. 2, pp. 131-135, 2012.

[56] P. Stall, Evaluation of patients with fibromyalgic syndrome treated by the Structural Integration Rolfing method and by acupuncture-abstract [Tese de Doutorado], University of Sao Paolo, Faculty of Medicine, São Paulo, Brazil, 2013, http://www.teses.usp.br/teses/disponiveis/5/5138/tde-09082013121202/pt-br.php.

[57] J. C. Licciardone, S. T. Stoll, K. G. Fulda et al., "Osteopathic manipulative treatment for chronic low back pain: a randomized controlled trial," Spine, vol. 28, no. 13, pp. 1355-1362, 2003.

[58] L. Niemistö, T. Lahtinen-Suopanki, P. Rissanen, K.-A. Lindgren, S. Sarna, and H. Hurri, "A randomized trial of combined manipulation, stabilizing exercises, and physician consultation compared to physician consultation alone for chronic low back pain," Spine, vol. 28, no. 19, pp. 2185-2191, 2003.

[59] D. Carnes, B. Mullinger, and M. Underwood, "Defining adverse events in manual therapies: a modified Delphi consensus study," Manual Therapy, vol. 15, no. 1, pp. 2-6, 2010.

[60] D. Carnes, T. S. Mars, B. Mullinger, R. Froud, and M. Underwood, "Adverse events and manual therapy: a systematic review," Manual Therapy, vol. 15, no. 4, pp. 355-363, 2010. 
[61] J. Licciardone, R. Gamber, and K. Cardarelli, "Patient satisfaction and clinical outcomes associated with osteopathic manipulative treatment," Journal of the American Osteopathic Association, vol. 102, no. 1, pp. 13-20, 2002.

[62] D. M. Eisenberg, D. E. Post, R. B. Davis et al., "Addition of choice of complementary therapies to usual care for acute low back pain: a randomized controlled trial," Spine, vol. 32, no. 2, pp. 151158, 2007.

[63] D. C. Cherkin, K. J. Sherman, J. Kahn et al., "A comparison of the effects of 2 types of massage and usual care on chronic low back pain: a randomized, controlled trial," Annals of Internal Medicine, vol. 155, no. 1, pp. 1-9, 2011.

[64] N. M. C. Alexandre, M. Nordin, R. Hiebert, and M. Campello, "Predictors of compliance with short-term treatment among patients with back pain," Pan American Journal of Public Health, vol. 12, no. 2, pp. 86-94, 2002. 


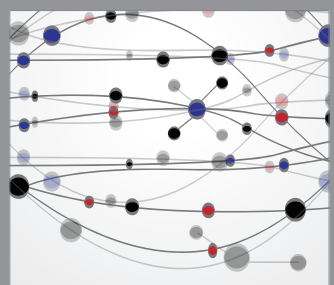

The Scientific World Journal
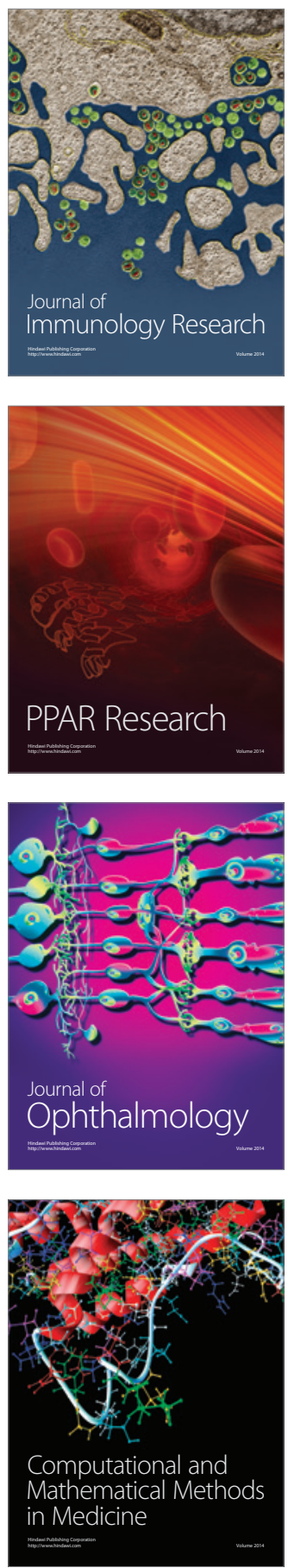

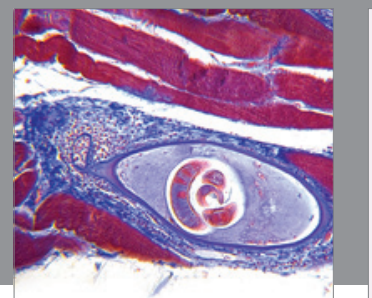

Gastroenterology

Research and Practice
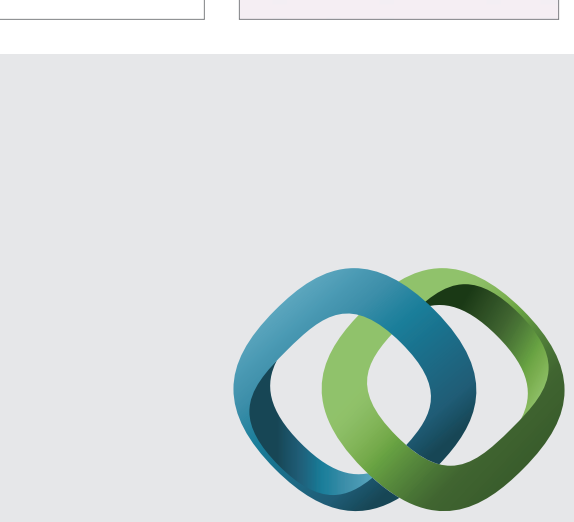

\section{Hindawi}

Submit your manuscripts at

http://www.hindawi.com
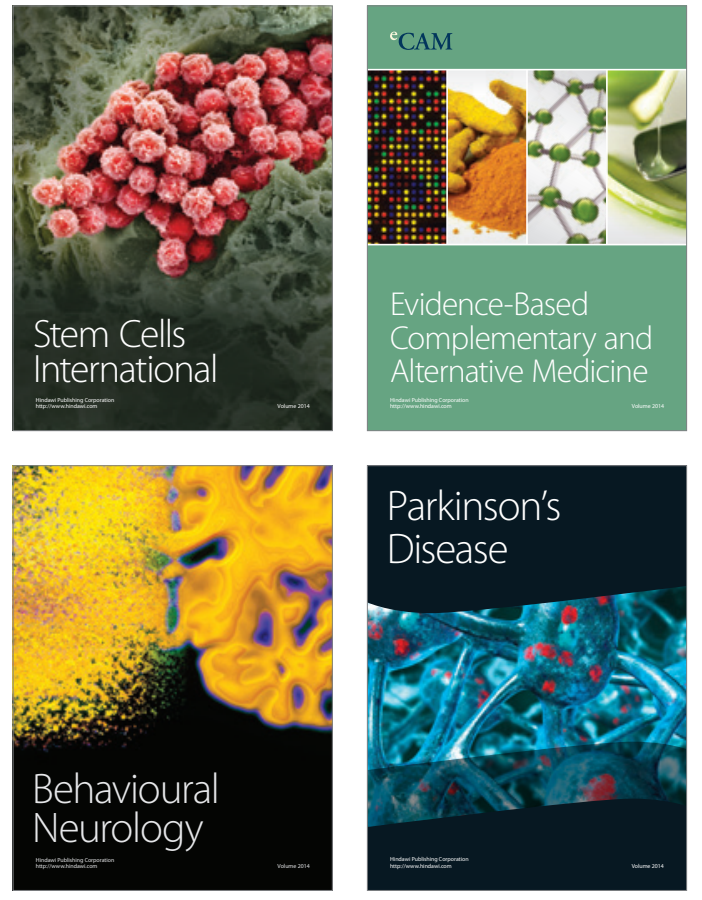
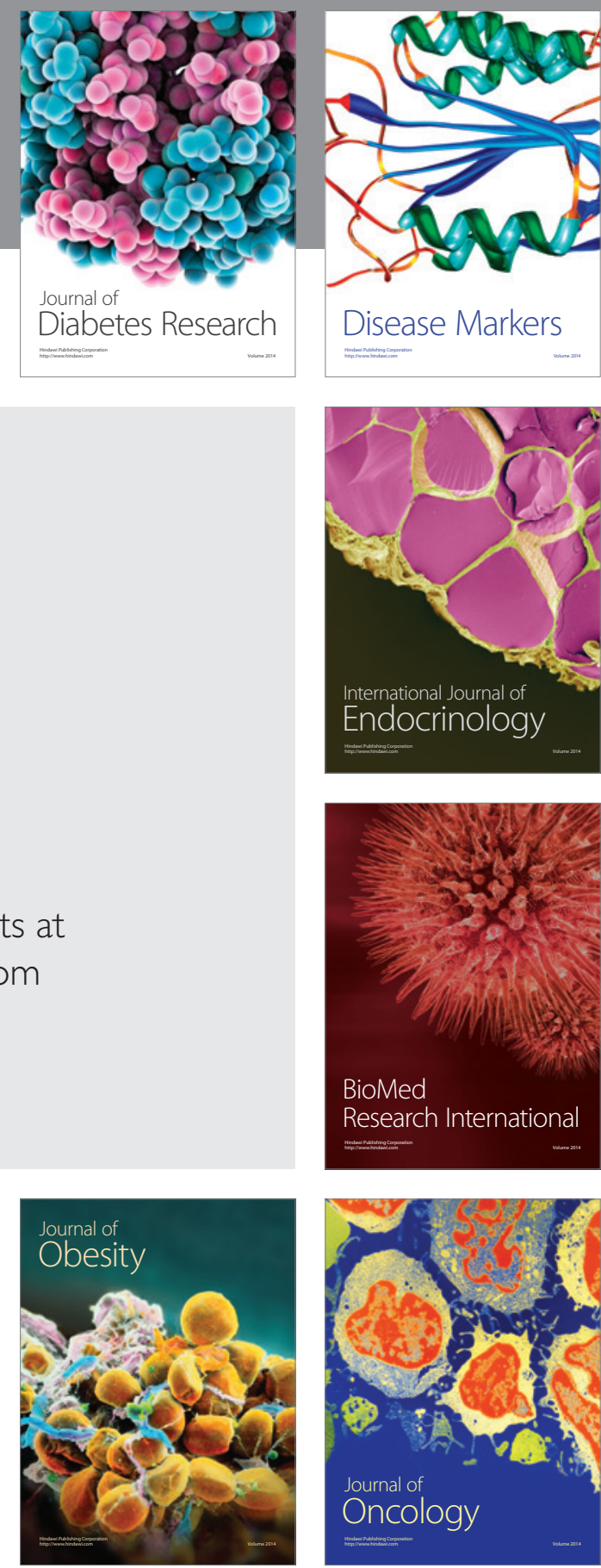

Disease Markers
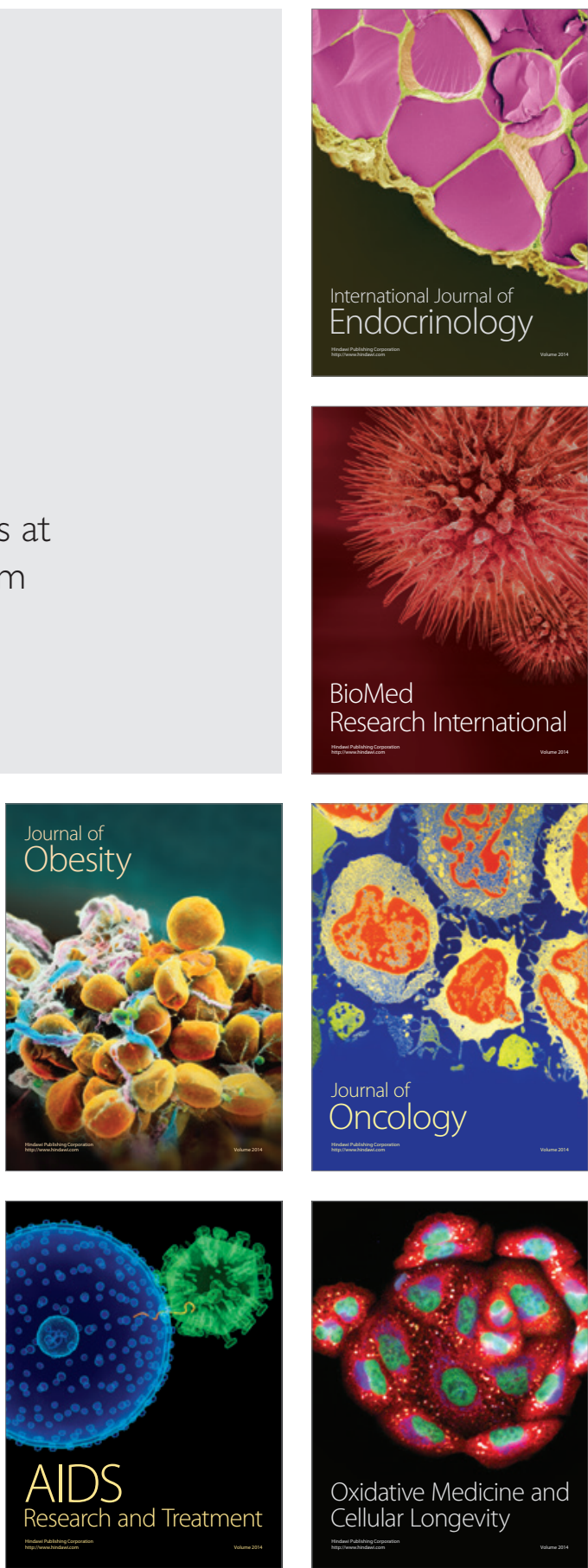